\title{
La dialisi peritoneale ovvero il gioco delle perle di vetro
}

\author{
Carlo Crepaldi
}

\author{
U.O. Nefrologia, Dialisi e Trapianto Renale, Ospedale Civile San Bortolo, Vicenza
}

\begin{abstract}
Peritoneal dialysis, or the glass bead game
Abstract. The prevalence of peritoneal dialysis did not increase in Italy during the last years. It remains still unclear why so many Italian Nephrology Units do not offer peritoneal dialysis as an efficient renal replacement therapy for patients with stage 5 chronic kidney disease. Hemodialysis and peritoneal dialysis should contribute to generate an integrated system in the same Nephrology Unit to tailor the best dialytic option for the uremic patient.
\end{abstract}

Key words: Peritoneal dialysis, Barriers to the diffusion of peritoneal dialysis, Choice of dialytic strategy

Conflict of interest: None.

Financial support: None.

Accettato: 17 Agosto 2013

Venti anni di dialisi extracorporea e di terapie sostitutive in area critica sono tanto tempo, il tempo di passare da giovane nefrologo a medico brizzolato. Pazienti, Infermieri e Colleghi sono arrivati, altri, in questa fetta di vita, hanno preso strade diverse, ma la collega Alessandra Brendolan e io sempre lì, in sala dialisi: conoscevamo più i pazienti che tanti nostri parenti. Venti anni in emodialisi vogliono anche dire passare dall'Unimat, dal bellissimo emofiltratore Sifra e dal Monitral all'“on-line", all'AFB, alla Physio e, poi, all'area critica... Insomma, non ricordo una mattina che sia andato al lavoro di malavoglia. Poi, un bel giorno, l'amico Roberto Dell'Aquila va altrove e la dialisi peritoneale viene affidata a me. L'avevo respirata sin da giovane assistente dal Prof. La Greca, da Claudio Ronco e da Mariano Feriani. Governare da responsabile una realtà così centrale e radicata a Vicenza era, però, un'altra cosa e, all'inizio, non è stato facile. I pazienti per me erano nuovi da gestire e dovevo cambiare radicalmente la mia prospettiva, ora orientata su pazienti più "ambulatoriali" e meno "cronicizzati". Gli Infermieri no, quelli li conoscevo bene, erano passati tutti dall'extracorporea prima, e li apprezzavo per come sapevano svolgere il loro difficile compito. Poi, giorno dopo giorno, vedo i pazienti: è pazzesco, stanno bene, hanno esami buonissimi, hanno diuresi oltre il litro anche dopo 2-3 anni di dialisi, vivono meglio, spesso lavorano e hanno meno sete e alcuni interagiscono e si confrontano con Medici e Infermieri su terapie e strategie. E, a casa, questi pazienti hanno un palo, un tavolino, uno scaldasacche e la forza di gravità oppure un cycler, non certo un complesso monitor per l'extracorporea. Ed è qui che ho scoperto la grandezza della dialisi peritoneale, nella genialità della sua semplicità. Vivendola da dentro, giorno dopo giorno, ti accorgi che la PD è una dialisi straordinaria per il paziente e per il nefrologo. Per me è uno stimolo personale, culturale e professionale impagabile. Ogni paziente è un puzzle dove devi combinare gli aspetti sociali e umani, il trasporto peritoneale e le opzioni dialitiche e farmacologiche. Una specie di gioco delle perle di vetro dove devi mettere tanto del tuo. Poi, vedi la prevalenza della PD in Italia: $10 \%$. Ma come? Ti dicono che in certi Centri non viene neppure proposta al paziente. Perché? Eppure, per tanti nefrologi, la PD non riveste alcun aspetto marginale. Occorre ringraziare, per questo, i Padri della metodica, da Ganter a Maxwell, da Moncrief a Oreopoulos, e le altre grandi figure che hanno consentito con il loro ingegno dei salti epocali nella tecnica dialitica peritoneale. Ma, ancora di più, vanno ricordati tutti i nefrologi che, senza riconoscimenti né vetrine, e sono tanti anche in Italia, dedicano da anni ogni giorno il loro tempo e le loro migliori risorse semplicemente per trattare al meglio, talvolta contro la corrente, i loro pazienti peritoneali. Perché, parafrasando e adattando al diverso contesto dei versi di Samuel Beckett: "I pazienti arrivano, uguali e diversi. Con ciascuno è uguale e diverso. Con ciascuno il rapporto è uguale, con ciascuno il rapporto è diverso". Anche questo, per me, è la PD. 


\section{Riassunto}

La dialisi peritoneale (PD) in Italia è un trattamento sostitutivo renale di nicchia, limitato numericamente nel numero di pazienti trattati e nel numero di Centri che lo propongono. Non esistono, allo stato attuale, valide motivazioni cliniche e dialitiche che facciano in generale preferire la dialisi extracorporea (HD) alla PD. Al contrario, la dialisi peritoneale potrebbe trovare ampio spazio come "first choi$c e$ " nei pazienti uremici incidenti a diuresi conservata per la sua validità nel preservare la funzione renale residua. Le due metodiche dialitiche (HD e PD) dovrebbero convivere e integrarsi nella stessa Unità Operativa, arricchendo la proposta dialitica al paziente. Compito del nefrologo dovrebbe essere, infatti, quello di offrire senza preconcetti e di confezionare il trattamento sostitutivo che meglio si adatta al paziente nella sua complessa realtà umana, clinica, ambientale e sociale.
Parole chiave: Dialisi peritoneale, Barriere alla diffusione della dialisi peritoneale, Scelta della metodica dialitica

Dichiarazione di conflitto di interessi: L'Autore dichiara di non avere conflitto di interessi.

Contributi economici degli autori: L'Autore dichiara di non avere rapporti anche di finanziamento con soggetti portatori di interessi commerciali in campo sanitario.

Indirizzo degli Autori:

Dr. Carlo Crepaldi

U.O. Nefrologia, Dialisi e Trapianto Renale

Ospedale Civile San Bortolo

36100 Vicenza

carlo.crepaldi@ulssvicenza.it 